\title{
The Role of Proteoglycans in Contributing to Placental Thrombosis and Fetal Growth Restriction
}

\author{
Joanne M. Said ${ }^{1,2}$ \\ ${ }^{1}$ Level 7 Research Precinct, The Royal Women's Hospital, Locked Bag 300, Parkville, VIC 3052, Australia \\ ${ }^{2}$ Department of Obstetrics and Gynaecology, The University of Melbourne, Parkville 3052, Australia
}

Correspondence should be addressed to Joanne M. Said, jo.said@thewomens.org.au

Received 21 November 2010; Accepted 13 January 2011

Academic Editor: Fabio Facchinetti

Copyright (C) 2011 Joanne M. Said. This is an open access article distributed under the Creative Commons Attribution License, which permits unrestricted use, distribution, and reproduction in any medium, provided the original work is properly cited.

\begin{abstract}
Fetal growth restriction is an important pregnancy complication that has major consequences for the fetus and neonate as well as an increased risk of long-term morbidity extending into adulthood. The precise aetiology of most cases of fetal growth restriction is unknown although placental thrombosis is a common feature in many of these cases. This paper will outline the potential role of proteoglycans in contributing to placental thrombosis and fetal growth restriction.
\end{abstract}

\section{Introduction}

Adverse pregnancy outcomes remain a major cause of perinatal and paediatric morbidity and mortality, and subsequent morbidity into adult life. One of the key causes of adverse pregnancy outcome is fetal growth restriction (FGR). Fetal growth restriction describes a situation in which the fetus fails to achieve its full growth potential in utero. While an aetiology can be ascribed in a number of cases, the precise aetiology remains uncertain in up to $70 \%$ of cases. The ability to predict "at risk" pregnancies is limited, and therapeutic strategies are largely restricted to ultrasound surveillance for evidence of fetal decompensation secondary to hypoxia and then timely intervention by delivery. There is a trade off between delivering too early with the resultant risks of prematurity and delaying delivery with the attendant risks associated with hypoxia, neurological damage, and fetal demise. Of further concern is the accumulating evidence that growth-restricted fetuses are at increased risk of longterm postnatal sequelae including obesity, type II diabetes, hypertension, cardiovascular disease, and premature death [1]. While a number of maternal and fetal factors have been identified which contribute to FGR, the aetiologies of the vast majority of cases remain uncertain [2]. The pathogenesis of FGR appears to be related to abnormalities in the placental vascular flow [3] possibly due to microvascular thrombosis within the placenta [4]. The importance of understanding the mechanisms that control coagulation within the placenta cannot be overemphasised. Dysregulation of coagulation within the placenta is one of the few mechanisms which may potentially be amenable to therapy via anticoagulant treatment (including the development of specific targeted anticoagulants), hence it is imperative that this hitherto unexplored area of placental function be rigorously investigated.

\section{Regulation of Thrombin within the Placenta}

Normal human pregnancy is characterised by increased thrombin generation resulting in a shift towards a hypercoaguable state [5]. Amongst factors responsible for this alteration in systemic haemostasis is the possibility that excessive thrombin production may originate in the placenta [6]. Despite this, the placentae of normal human pregnancies are not characterized by excessive intravascular thrombosis, suggesting that factors within the placenta itself may offset the trend toward hypercoaguability within this organ [6]. In contrast, excessive uteroplacental thrombosis has been proposed as the pathogenic mechanism leading to a range of pregnancy complications including fetal growth restriction $[4,7,8]$. Histopathologic assessment of placentae from a range of pregnancy complications has demonstrated the presence of mural thrombi within both the maternal and fetal compartments of the placenta [9-12]. 
It is likely that the coagulation disturbance seen with FGR arises within the placenta itself rather than within the systemic circulation of either the fetus or the mother. Despite early work suggesting associations between inherited thrombophilias and adverse pregnancy outcomes $[13,14]$, numerous case-control studies $[15,16]$ and prospective cohort studies [17-19] examining maternal thrombophilias have failed to confirm significant associations between FGR and thrombophilias. Furthermore, recent prospective cohort studies have suggested that most patients with thrombophilias do not develop pregnancy complications and that most patients who do develop these complications do not carry a thrombophilic marker [17-19]. Likewise, several studies investigating the possible contribution of fetal or neonatal inherited thrombophilias to adverse pregnancy outcomes have failed to confirm this association $[15,16]$. While it remains possible that the placental thrombosis that is observed in patients with these adverse pregnancy outcomes may be due to other (as yet unidentified) thrombophilic markers, a more likely explanation is that the coagulation disturbance arises within the placenta itself.

\section{Proteoglycans}

Proteoglycans are macromolecules located within vessel walls that contain a core protein to which sulphated glycosaminoglycan (GAG) chains are covalently linked. There are four types of GAG chains located in the blood vessel wall: chondroitin sulphate, dermatan sulphate, heparan sulphate, and hyaluronan [20]. However, only chondroitin sulphate, dermatan sulphate, and heparin sulphate are covalently linked to a core protein [20].

Placentae contain two major types of proteoglycans; those containing heparan sulphate and those containing chondroitin sulphate or dermatan sulphate [21]. Heparan sulphate proteoglycans in the placenta include syndecan and perlecan $[22,23]$ while chondroitin sulphate and dermatan sulphate containing proteoglycans in the placenta include decorin and biglycan [24]. Decorin usually has one chondroitin sulphate or dermatan sulphate GAG attached to its core protein, while biglycan normally has two of these GAGs attached to its core.

Swan et al. have previously demonstrated that decorin is localised to the stroma surrounding fetal blood vessels of placental villi [25], whereas Murthi et al. demonstrated that biglycan is localized to endothelial and smooth muscle cells of fetal capillaries [26]. In contrast, syndecan 1 was highly expressed in the syncytiotrophoblast [27].

\section{Actions of Proteoglycans and GAGs in the Human Placenta}

Proteoglycans and their GAG sidechains have been shown to have important actions within the placenta. These molecules display important anticoagulant actions which suggest they may play a key role in preventing thrombotic events within the placenta $[6,28]$. Heparan sulphate chains bind Antithrombin through a pentasaccharide sequence, while dermatan sulphate chains bind heparin cofactor II (HCII) through a highly charged sequence [28] GAG bound antithrombin and HCII are thought to undergo a conformational change, which in turn facilitates the inhibition of thrombin $[29,30]$. The cellular localization of PGs to endothelium and cells in contact with circulating blood, suggest an important role for these molecules in localized anticoagulation.

\section{Could Proteoglycans Contribute to FGR?}

Swan et al. demonstrated a significant reduction in the mRNA and protein expression of decorin in placentae from human pregnancies complicated by FGR compared to gestation matched controls [25]. Likewise, Murthi et al. demonstrated a significant reduction in the mRNA and protein expression of biglycan in FGR compared to gestation matched controls. Both studies confirmed the localization of these molecules in close proximity to circulating blood, supporting the hypothesis that localized anticoagulant activity may be reduced in FGR placentae. Whether these changes correlate with the increased placental thrombosis observed in FGR placentae remains uncertain. Likewise, it is plausible that the pathophysiological process resulting in FGR is not purely restricted to alterations in thrombin regulation, since decorin has been shown to interact with insulin-like growth factor-1 [31], thereby raising the possibility that altered decorin expression may interfere with growth factor expression and hence fetal growth. Decorin has also been shown to inhibit trophoblast proliferation and migration $[32,33]$ raising yet another potential mechanism by which altered decorin expression may play a role in the pathogenesis of fetal growth restriction.

Fetal growth restriction is commonly associated with pre-eclampsia and hypertensive disorders of pregnancy [2]. Altered GAG sulphation patterns have been observed in placentae from pregnancies complicated by pre-eclampsia [34], raising the possibility that this could result in altered GAG function and thereby contribute to the development of pre-eclampsia. Chen et al. have also demonstrated upregulation of proteoglycan expression and alterations in the GAG composition in placentae from women with gestational diabetes and hyperglycaemia [24]. Gestational diabetes is typically associated with fetal overgrowth; however, increased fetal morbidity and mortality are well recognized [35]. It is plausible that altered placental proteoglycan homeostasis may be an important mechanism contributing to the poorer perinatal outcomes observed in all these pregnancy complications.

However, determining causation is not always easy in human pregnancy research so it remains uncertain whether the alterations in proteoglycan expression observed are truly causative, or rather reflect a response to an altered growth process. Ishiguro et al. has demonstrated alterations in placental vasculature in Syndecan 4 knockout mice with greater evidence of fibrin and calcium deposition suggesting disruption of the local anticoagulant mechanisms, although a statistically significant growth disturbance was not seen [36]. 


\section{Potential Therapies}

At present, there are no proven treatments for FGR. Understanding the potential role of proteoglycans in contributing to FGR is important as it may allow the rational use of therapeutic glycosaminoglycans (i.e., heparins) to augment the anticoagulant activities of proteoglycans. The challenge, however, is to ensure that this anticoagulant activity is delivered to the compartment of the placenta in which the functional defect can be demonstrated and that undesired effects of systemic anticoagulation for either mother or fetus can be avoided.

\section{Conclusion}

Fetal growth restriction is a serious pregnancy complication that cannot currently be "treated" during pregnancy. Management relies on appropriate detection and timely intervention by delivery to prevent the serious consequences of this condition. This often necessitates the delivery of extremely premature infants who require the investment of a considerable range of financial, intellectual, and emotional resources to ensure their survival. Furthermore, these infants are at risk of developing the long-term consequences of these conditions which may not become apparent until adulthood such as an increased risk of obesity, cardiovascular disease, and diabetes. Understanding the pathophysiological role of proteoglycans in the placenta may provide an important therapeutic window to prevent or treat fetal growth restriction in the future. There is no doubt that efforts to improve the fetal and neonatal outcomes for these infants appear well justified given the possible life-long improvements which may ensue.

\section{References}

[1] D. J. P. Barker, P. D. Gluckman, K. M. Godfrey, J. E. Harding, J. A. Owens, and J. S. Robinson, "Fetal nutrition and cardiovascular disease in adult life," The Lancet, vol. 341, no. 8850, pp. 938-941, 1993.

[2] D. Maulik, "Fetal growth restriction: the etiology," Clinical Obstetrics and Gynecology, vol. 49, no. 2, pp. 228-235, 2006.

[3] D. Maulik, J. Frances Evans, and L. Ragolia, "Fetal growth restriction: pathogenic mechanisms," Clinical Obstetrics and Gynecology, vol. 49, no. 2, pp. 219-227, 2006.

[4] M. Sugimura, R. Ohashi, T. Kobayashi, and N. Kanayama, "Intraplacental coagulation in intrauterine growth restriction: cause or result?" Seminars in Thrombosis and Hemostasis, vol. 27, no. 2, pp. 107-114, 2001.

[5] F. R. Gerbasi, S. Bottoms, A. Farag, and E. Mammen, "Increased intravascular coagulation associated with pregnancy," Obstetrics and Gynecology, vol. 75, no. 3 I, pp. 385-389, 1990.

[6] M. A. Delorme, L. Xu, L. Berry, L. Mitchell, and M. Andrew, "Anticoagulant dermatan sulfate proteoglycan (decorin) in the term human placenta," Thrombosis Research, vol. 90, no. 4, pp. 147-153, 1998.

[7] J. R. Higgins and S. P. Brennecke, "Pre-eclampsia-still a disease of theories?" Current Opinion in Obstetrics and Gynecology, vol. 10, no. 2, pp. 129-133, 1998.
[8] N. Lanir, A. Aharon, and B. Brenner, "Haemostatic mechanisms in human placenta," Best Practice and Research: Clinical Haematology, vol. 16, no. 2, pp. 183-195, 2003.

[9] C. M. Salafia, V. K. Minior, J. C. Pezzullo, E. J. Popek, T. S. Rosenkrantz, and A. M. Vintzileos, "Intrauterine growth restriction in infants of less than thirty-two weeks' gestation: associated placental pathologic features," American Journal of Obstetrics and Gynecology, vol. 173, no. 4, pp. 1049-1057, 1995.

[10] C. M. Salafia, J. C. Pezzullo, J. A. López-Zeno, S. Simmens, V. K. Minior, and A. M. Vintzileos, "Placental pathologic features of preterm preeclampsia," American Journal of Obstetrics and Gynecology, vol. 173, no. 4, pp. 1097-1105, 1995.

[11] R. W. Redline and A. Pappin, "Fetal thrombotic vasculopathy: the clinical significance of extensive avascular villi," Human Pathology, vol. 26, no. 1, pp. 80-85, 1995.

[12] C. M. Salafia, J. C. Pezzullo, V. K. Minior, and M. Y. Divon, "Placental pathology of absent and reversed end-diastolic flow in growth- restricted fetuses," Obstetrics and Gynecology, vol. 90, no. 5, pp. 830-836, 1997.

[13] Z. Alfirevic, D. Roberts, and V. Martlew, "How strong is the association between maternal thrombophilia and adverse pregnancy outcome? A systematic review," European Journal of Obstetrics Gynecology and Reproductive Biology, vol. 101, no. 1, pp. 6-14, 2002.

[14] L. Robertson, O. Wu, P. Langhorne et al., "Thrombophilia in pregnancy: a systematic review," British Journal of Haematology, vol. 132, no. 2, pp. 171-196, 2006.

[15] C. Infante-Rivard, G. E. Rivard, W. V. Yotov et al., "Absence of association of thrombophilia polymorphisms with intrauterine growth restriction," New England Journal of Medicine, vol. 347, no. 1, pp. 19-25, 2002.

[16] J. C. Livingston, J. R. Barton, V. Park, B. Haddad, O. Phillips, and B. M. Sibai, "Maternal and fetal inherited thrombophilias are not related to the development of severe preeclampsia," American Journal of Obstetrics and Gynecology, vol. 185, no. 1, pp. 153-157, 2001.

[17] J. M. Said, J. R. Higgins, E. K. Moses et al., "Inherited thrombophilia polymorphisms and pregnancy outcomes in nulliparous women," Obstetrics and Gynecology, vol. 115, no. 1, pp. 5-13, 2010.

[18] D. Dizon-Townson, C. Miller, B. Sibai et al., "The relationship of the factor $\mathrm{V}$ Leiden mutation and pregnancy outcomes for mother and fetus," Obstetrics and Gynecology, vol. 106, no. 3, pp. 517-524, 2005.

[19] R. P. Murphy, C. Donoghue, R. J. Nallen et al., "Prospective evaluation of the risk conferred by factor $\mathrm{V}$ Leiden and thermolabile methylenetetrahydrofolate reductase polymorphisms in pregnancy," Arteriosclerosis, Thrombosis, and Vascular Biology, vol. 20, no. 1, pp. 266-270, 2000.

[20] R. V. Iozzo, "Basement membrane proteoglycans: from cellar to ceiling," Nature Reviews Molecular Cell Biology, vol. 6, no. 8, pp. 646-656, 2005.

[21] R. N. Achur, M. Valiyaveettil, A. Alkhalil, C. F. Ockenhouse, and D. C. Gowda, "Characterization of proteoglycans of human placenta and identification of unique chondroitin sulfate proteoglycans of the intervillous spaces that mediate the adherence of Plasmodium falciparum-infected erythrocytes to the placenta," Journal of Biological Chemistry, vol. 275, no. 51, pp. 40344-40356, 2000.

[22] V. Jokimaa, P. Inki, H. Kujari, O. Hirvonen, E. Ekholm, and L. Anttila, "Expression of syndecan-1 in human placenta and decidua," Placenta, vol. 19, no. 2-3, pp. 157-163, 1998. 
[23] W. C. V. Yang, T. H. Su, Y. C. Yang, S. C. Chang, C. Y. Chen, and C. P. Chen, "Altered perlecan expression in placental development and gestational diabetes mellitus," Placenta, vol. 26, no. 10, pp. 780-788, 2005.

[24] C. P. Chen, S. C. Chang, and W. C. Vivian Yang, "High glucose alters proteoglycan expression and the glycosaminoglycan composition in placentas of women with gestational diabetes mellitus and in cultured trophoblasts," Placenta, vol. 28, no. 2-3, pp. 97-106, 2007.

[25] B. C. Swan, P. Murthi, G. Rajaraman et al., "Decorin expression is decreased in human idiopathic fetal growth restriction," Reproduction, Fertility and Development, vol. 22, no. 6, pp. 949-955, 2010.

[26] P. Murthi, F. A. Faisal, G. Rajaraman et al., "Placental Biglycan Expression is Decreased in Human Idiopathic Fetal Growth Restriction," Placenta, vol. 30, no. 9, p. A.70, 2009.

[27] G. Rajaraman, "Placental Syndecan-1 expression is significantly reduced in idiopathic fetal growth restriction," Placenta, vol. 30, no. 9, p. A.72, 2009.

[28] T. K. Giri and D. M. Tollefsen, "Placental dermatan sulfate: isolation, anticoagulant activity, and association with heparin cofactor II," Blood, vol. 107, no. 7, pp. 2753-2758, 2006.

[29] J. Chen and J. Liu, "Characterization of the structure of antithrombin-binding heparan sulfate generated by heparan sulfate 3-O-sulfotransferase 5," Biochimica et Biophysica Acta, vol. 1725, no. 2, pp. 190-200, 2005.

[30] S. Brinkmeyer, R. Eckert, and H. Ragg, "Reformable intramolecular cross-linking of the N-terminal domain of heparin cofactor II: effects on enzyme inhibition," European Journal of Biochemistry, vol. 271, no. 21, pp. 4275-4283, 2004.

[31] E. Schönherr, C. Sunderkötter, R. V. Iozzo, and L. Schaefer, "Decorin, a novel player in the insulin-like growth factor system," Journal of Biological Chemistry, vol. 280, no. 16, pp. 15767-15772, 2005.

[32] D. Iacob, J. Cai, M. Tsonis et al., "Decorin-mediated inhibition of proliferation and migration of the human trophoblast via different tyrosine kinase receptors," Endocrinology, vol. 149, no. 12, pp. 6187-6197, 2008.

[33] G. Xu, M. J. Guimond, C. Chakraborty, and P. K. Lala, "Control of proliferation, migration, and invasiveness of human extravillous trophoblast by decorin, a decidual product," Biology of Reproduction, vol. 67, no. 2, pp. 681-689, 2002.

[34] M. Warda, F. Zhang, M. Radwan et al., "Is human placenta proteoglycan remodeling involved in pre-eclampsia?" Glycoconjugate Journal, vol. 25, no. 5, pp. 441-450, 2008.

[35] The HAPO Study Cooperative Research Group, "Hyperglycemia and adverse pregnancy outcomes," The New England Journal of Medicine, vol. 358, no. 19, pp. 1991-2002, 2008.

[36] K. Ishiguro, K. Kadomatsu, T. Kojima et al., "Syndecan-4 deficiency impairs the fetal vessels in the placental labyrinth," Developmental Dynamics, vol. 219, no. 4, pp. 539-544, 2000. 


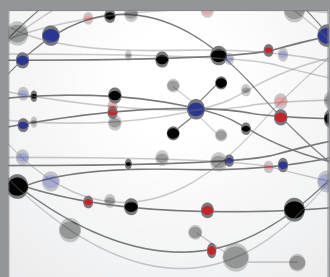

The Scientific World Journal
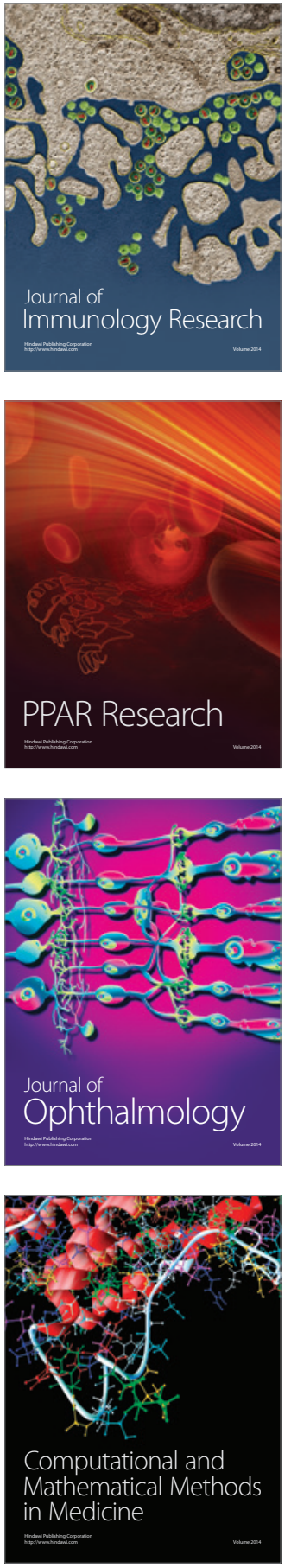

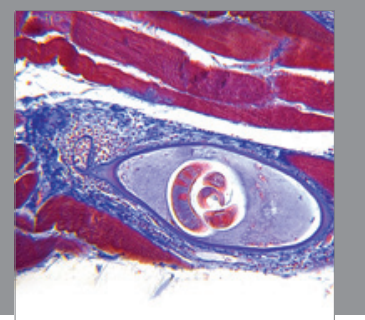

Gastroenterology

Research and Practice
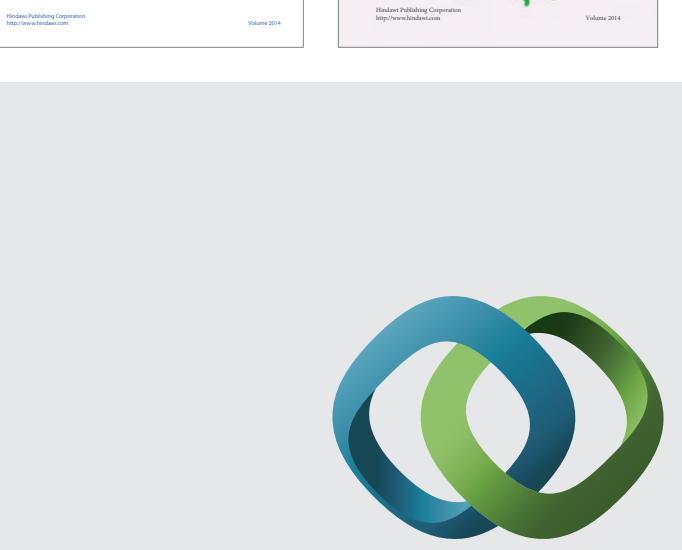

\section{Hindawi}

Submit your manuscripts at

http://www.hindawi.com
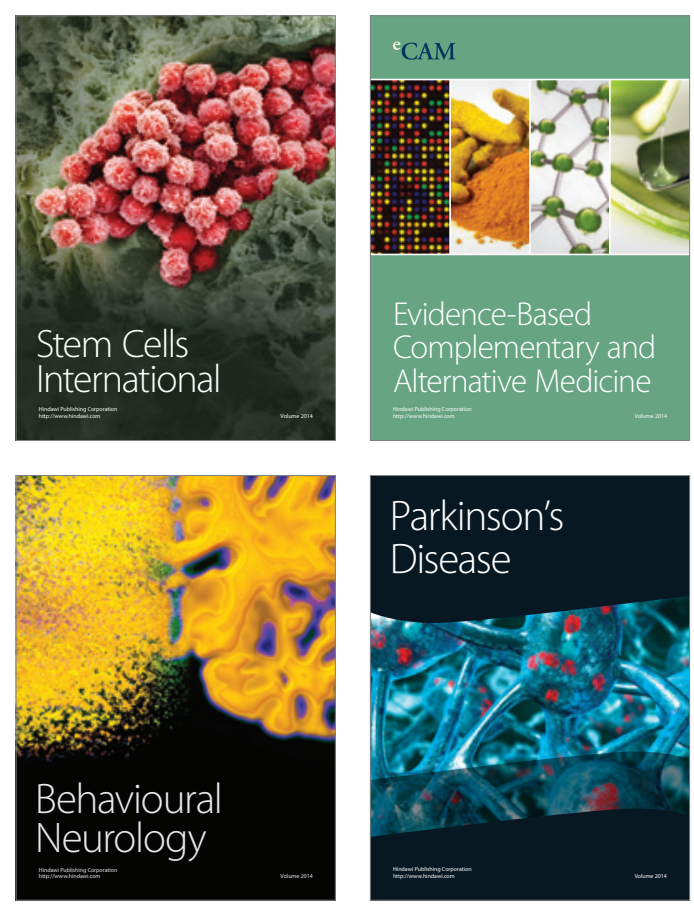

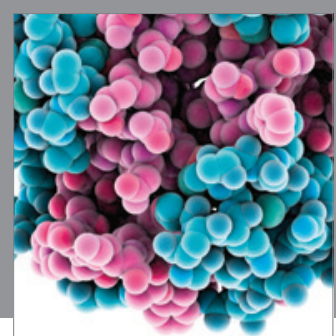

Journal of
Diabetes Research

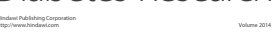

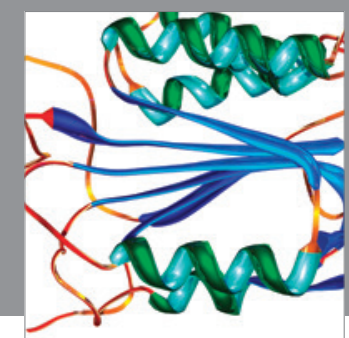

Disease Markers
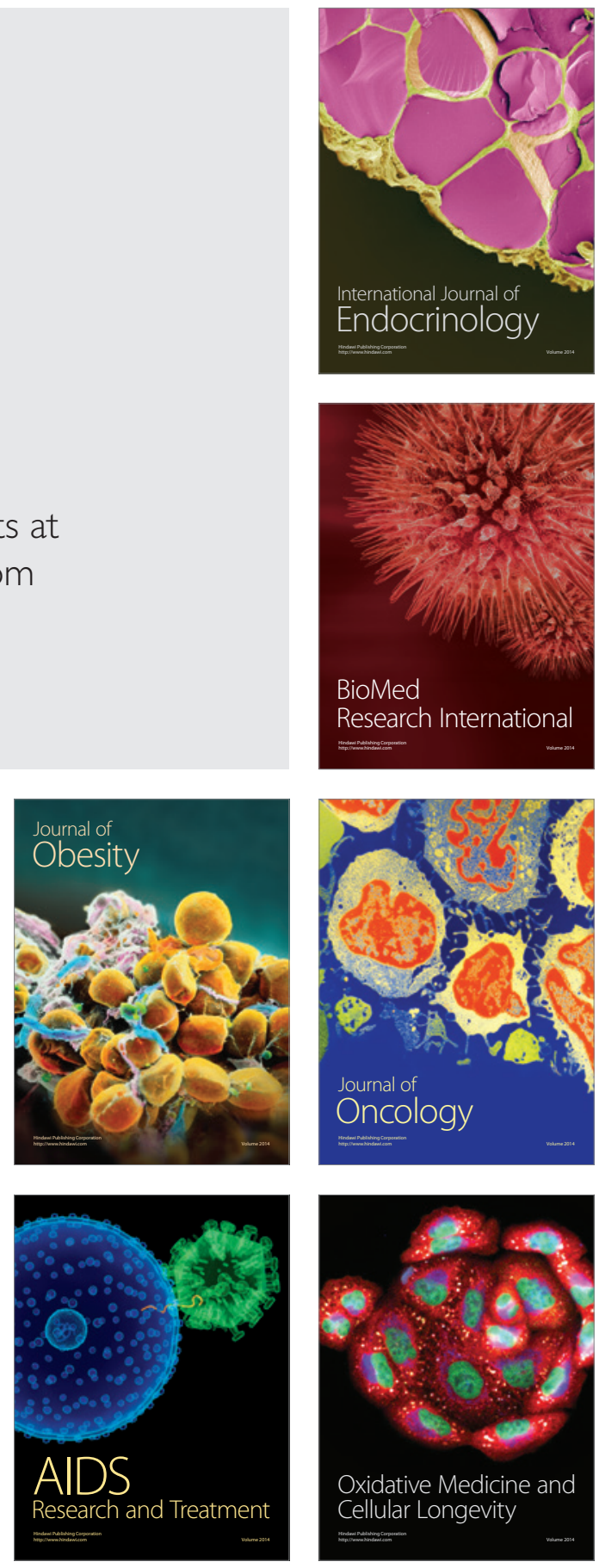